\title{
Práticas Educativas Parentais e Problemas Emocionais/Comportamentais em Adolescentes com Altas Habilidades/Superdotação Intelectivas
}

\author{
Erica Isabel Dellatorre Andrade ${ }^{1}$ \\ ${ }^{1}$ Universidade do Vale do Rio dos Sinos, \\ RS, Brasil. \\ Jade Wagner Bernardes ${ }^{2}$ \\ ${ }^{2}$ Universidade Federal do Rio Grande do Sul, \\ RS, Brasil.
}

\author{
Carolina Macedo de Saraiva Lisboa ${ }^{3}$ \\ ${ }^{3}$ Pontifícia Universidade Católica do Rio Grande do Sul, \\ RS, Brasil. \\ Angela Helena Marin ${ }^{2}$ \\ ${ }^{2}$ Universidade Federal do Rio Grande do Sul, \\ RS, Brasil.
}

Resumo: Este estudo teve como objetivo analisar a associação entre práticas educativas parentais e problemas emocionais de comportamento em adolescentes com altas habilidades/ superdotação (AH/SD). Buscou-se também verificar o poder preditivo das variáveis sociodemográficas e das práticas educativas parentais percebidas em relação aos problemas emocionais e de comportamento, além de investigar diferenças entre os sexos. Trata-se de um estudo de delineamento observacional e explicativo, com corte transversal, do qual participaram 14 adolescentes com AH/SD intelectiva, aferidos pela Escala de Inteligência Wechsler Abreviada (WASI), sendo 10 meninos $(71,4 \%)$ e quatro meninas $(28,6 \%)$ com idade entre 12 e 16 anos $(\mathrm{M}=13,2$; $\mathrm{DP}=1,4)$. Todos responderam ao Questionário de Dados Sociodemográficos, às Escalas de Práticas Parentais e ao Inventário de Comportamentos Autorreferidos para jovens de 11 a 18 anos. Os resultados indicaram predominância de práticas educativas maternas, como cobrança de responsabilidade, apoio emocional e incentivo à autonomia. Os meninos apresentaram mais indicadores de problemas internalizantes e total de problemas, superando em cerca de $30 \%$ a média das meninas. O total de problemas identificados foi explicado em $17,4 \%$ pelo menor incentivo à autonomia por parte da mãe. Por outro lado, os indicadores de aspectos positivos foram explicados em $24,9 \%$ pela menor presença de controle punitivo, também da mãe. Os dados apontam para uma maior incidência de indicadores de problemas emocionais e de comportamento em meninos com AH/SD e sua associação com características da família, especialmente as práticas educativas maternas, sugerindo a importância de atentar-se para tais questões.

Palavras-chave: Superdotados, Práticas Educativas, Problemas Emocionais, Problemas de Comportamento.

\section{Parental Educational Practices and Emotional/Behavioral Problems in High-Ability/Gifted Adolescents}

\begin{abstract}
This study aimed to examine the association between parental educational practices and emotional/behavioral problems in adolescents with high abilities/giftedness (HA/G), besides verifying the predictive power of these practices and sociodemographic variables on emotional and behavioral problems and differences between genders. This is a cross-sectional, observational and explanatory study conducted with 14 adolescents aged between 12 and 16 years $(\mathrm{M}=13.2, \mathrm{SD}=1.4)$, of whom 10 were boys $(71.4 \%)$ and four girls $(28.6 \%)$, considered as presenting with HA/G according to the Wechsler Intelligence Abbreviated Scale (WASI). Data were collected using the sociodemographic variables questionnaire, the Parental Practice
\end{abstract}


Scales, and the Youth Self-Report Behavior Inventory for Age 11-18. The results indicate a predominance of maternal educational practices such as responsibility demands, emotional support, and autonomy encouragement. Boys presented more internalizing problems indicators and total score problems about $30 \%$ higher than the mean value found for girls. Lower maternal autonomy encouragement explained $17.4 \%$ of the total problems identified. On the other hand, lower maternal punitive control explained $24.9 \%$ of positive aspects indicators. These data indicate that HA/G boys present a higher incidence of indicators of emotional and behavioral problems and that these problems are associated with family characteristics, especially maternal educational practices, point to the need for further looks at such issues.

Keywords: Gifted, Educational Practices, Emotional Problems, Behavior Problems.

\title{
Las Prácticas Educativas Parentales y los Problemas Emocionales/ Comportamentales en Adolescentes con Altas Habilidades/Superdotados
}

\begin{abstract}
Resumen: Este estudio pretendió identificar la relación entre las prácticas educativas parentales y los problemas emocionales y de comportamiento de adolescentes con altas habilidades/ superdotados (AH/SD). También se verificó el poder predictivo delas variables sociodemográficas y de las prácticas educativas parentales percibidas en relación a los problemas emocionales y de comportamiento, además de las diferencias entre los sexos. Este es un estudio observacional y explicativo, con cohorte transversal, en el que participaron 14 adolescentes con AH/SD evaluados por la Escala de Inteligencia Wechsler Abreviada (WASI), siendo 10 niños $(71,4 \%$ y cuatro niñas (28,6\%), con edades de entre 12 y 16 años $(\mathrm{M}=13,2$, $\mathrm{DE}=1,4)$. Todos respondieron al Cuestionario de Datos Sociodemográficos, a las Escalas de Prácticas Parentales y al Inventario de Comportamientos Autorreferidos para jóvenes de 11 a 18 años. Los resultados indicaron predominio de las prácticas educativas maternas, como cobranza de responsabilidad, apoyo emocional e incentivo a la autonomía. Los niños presentaron más indicadores de problemas internalizantes y del total de problemas, superando cerca del $30 \%$ la media de las niñas. El total de problemas identificados fue explicado en el $17,4 \%$ por el menor incentivo a la autonomía por parte de la madre. Por otro lado, los indicadores de aspectos positivos fueron explicados en el $24,9 \%$ por la menor presencia de control punitivo también por la madre. Los datos muestran una mayor incidencia de indicadores de problemas emocionales y de comportamiento en niños con AH/SD y su asociación con características de la familia, especialmente las prácticas educativas maternas, lo que evidencia la importancia de considerar estas cuestiones.

Palabras clave: Superdotados, Prácticas Educativas, Problemas Emocionales, Problemas de Comportamiento.
\end{abstract}

\section{Introdução}

Indivíduos com altas habilidades/superdotação (AH/SD) são aqueles que "apresentam um potencial elevado e grande envolvimento com as áreas do conhecimento humano, isoladas ou combinadas: intelectual, liderança, psicomotora, artes e criatividade" (Ministério da Educação, 2009, p. 2). No Brasil, até meados de 2005, o número de pessoas identificadas com AH/SD era menor do que 2 mil. Em 2018, no entanto, divulgou-se que havia 22.161 alunos com habilidades avançadas (Instituto Nacional de Estudos e Pesquisas Educacionais Anísio Teixeira, 2019). Esse aumento no número de identificação de casos se deveu ao trabalho dos Núcleos de Atividades de AH/SD (NAAH/SD), instituídos pelo Governo Federal em vários estados, que objetivavam identificar e promover o desenvolvimento das pessoas com $\mathrm{AH} / \mathrm{SD}$ (Ministério da Saúde, 2005), o que culminou na criação do Cadastro Nacional de Alunos com AH/SD, em 2015 (Lei n. 13.234, 2015). 
Embora seja crescente o número de jovens com $\mathrm{AH} / \mathrm{SD}$, seu entendimento e avaliação ainda requerem investimento. Mesmo que muito já se tenha avançado no estudo do tema, há, segundo Hakin (2016), carência de informações, especialmente quanto a questões relativas à família e sua associação com o desenvolvimento socioemocional de adolescentes (Pilarinos \& Solomon, 2017). Alguns estudos indicam que adolescentes com AH/SD podem apresentar uma percepção intelectual, socioemocional e comportamental diferente durante seu processo de desenvolvimento e aquisição de informações, o que pode levar a um antagonismo entre seus interesses e as exigências do ambiente (Alencar, 2007b; Dalosto \& Alencar, 2013; Ourofino \& Guimarães, 2007), despertando ansiedade, elevada autocobrança e tendência ao perfeccionismo (Dalosto \& Alencar, 2013; Piske, 2013). Tais sentimentos propiciam quadros de instabilidade emocional, depressão, baixa autoestima e dificuldades em estabelecer e manter relações sociais (Ourofino \& Fleith, 2011; Ourofino \& Guimarães, 2007), o que, por sua vez, pode levar ao isolamento, impactando o processo de escolarização, prejudicando o desenvolvimento de habilidades sociais e deixando a pessoa mais vulnerável a sentimentos de exposição e vergonha (Uzunian \& Vitalle, 2015).

Fugatea e Gentryb (2016) avaliaram os mecanismos usados para enfrentar as pressões acadêmicas em cinco meninas superdotadas e indicaram que, algumas vezes, a escola foi associada a sentimentos de confusão, tensão e vergonha, resultando em baixa autoestima e motivação, além de identificarem sentimento de frustração quando não alcançavam as expectativas dos pais. Os estudos sobre problemas emocionais e de comportamento em indivíduos com AH/SD não apresentam consenso quanto à maior ou menor incidência quando comparados à população em geral. Empiricamente, Gallucci, Middleton e Kline (1999), ao avaliarem 78 crianças superdotadas intelectualmente, constataram que foi insignificante a presença de problemas emocionais e de comportamento. Garland e Zigler (1999) indicaram, na avaliação de 191 jovens intelectualmente superdotados, que os mais talentosos do grupo tendiam a apresentar menos problemas emocionais e de comportamento do que os moderadamente dotados, assim como Richards, Encel e Shute (2003), que compararam 33 adolescentes com AH/SD intelectiva com 25 adolescentes com capacidade intelectual média e destacaram que os primeiros tinham níveis mais baixos de comportamento problemático.
Nessa mesma direção, Jones (2013) realizou uma revisão sistemática sobre crianças e jovens talentosos e identificou estudos que destacavam suas pontuações mais elevadas em medidas de saúde mental e bem-estar psicológico. Ademais, o estudo de Piske (2016) apontou que eles, em geral, têm autoestima elevada e conseguem lidar com críticas, além de, muitas vezes, segundo França-Freitas, Del Prette e Del Prette (2014), apresentarem um bom repertório de habilidades sociais.

Por outro lado, há estudos que sinalizam que indivíduos com $\mathrm{AH} / \mathrm{SD}$ apresentam maior propensão ao desenvolvimento de problemas emocionais e de comportamento. De modo particular, destacam-se como fatores de risco para indivíduos talentosos o desenvolvimento dessincrônico, o perfeccionismo e a ansiedade (Probst \& Piechowski, 2012; Silverman, 2002). O desenvolvimento dessincrônico se refere à combinação entre alto potencial cognitivo e alta intensidade emocional, que leva o indivíduo a ter uma autopercepção de que é qualitativamente diferente da norma (Silverman, 2002). Tal vivência pode provocar ansiedade em superdotados, uma vez que são capazes de perceber problemas em seus contextos, mas nem sempre conseguem resolvê-los (Neihart, 2002). A dessincronia também ocorre entre o desenvolvimento cognitivo e o físico, o que acarreta diferentes necessidades emocionais e sociais em crianças e adolescentes com AH/SD, podendo suscitar problemas emocionais e de comportamento (Terrassier, 2009).

Outro aspecto em relação aos jovens talentosos diz respeito à regulação emocional, pois, conforme Chung et al. (2011), eles tendem a apresentar maior dificuldade no processamento afetivo quando comparados a jovens com níveis medianos de inteligência. Consequentemente, é possível que crianças e adolescentes com AH/SD sofram maior rejeição entre pares, o que pode lhes fazer focar ainda mais em seu desenvolvimento intelectual e menos no desenvolvimento de habilidades sociais e de relacionamento (Cross \& Cross, 2015; Semrud-Clikeman, 2007). O perfeccionismo direcionado a si e ao outro também pode afetar a construção de relações interpessoais positivas (Stoeber, 2014). A dificuldade relacional tem sido indicada como produtora de ansiedade, podendo levar à desesperança e à depressão devido aos sentimentos de incompreensão e ameaça (Cross \& Cross, 2015). Eren, Çete, Avcil e Baykara (2018), em revisão de literatura, constataram que indivíduos 
talentosos apresentavam maiores escores em ansiedade, somatização e sintomas depressivos, bem como a incidência de déficit de atenção e hiperatividade.

Entre adolescentes com AH/SD também parece haver diferenças de acordo com o sexo. Ogurlu, Sevgi-Yalın e Yavuz-Birben (2016) revelaram que as meninas com AH/SD apresentaram médias significativamente maiores nas percepções de habilidades socioemocionais e de suporte social quando comparadas aos meninos. Já os meninos com AH/SD foram identificados como apresentando mais sintomas depressivos quando comparados a meninas talentosas (Eren et al., 2018).

Frente à heterogeneidade da população com AH/SD (Shaywitz et al., 2001), justifica-se a necessidade de dar continuidade à investigação sobre a incidência de problemas emocionais e de comportamento em adolescentes com AH/SD, associando-os com outras variáveis, como as de ordem familiar, entre elas, as práticas educativas parentais, definidas como estratégias utilizadas para educar os filhos que objetivam criar habilidades intelectuais, sociais e emocionais, bem como prevenir e extinguir comportamentos inadequados (Sapienza, Aznar-Farias, \& Silvares, 2009).

Alguns autores entendem que as práticas educativas coercitivas, assim como na população geral, podem levar crianças e adolescentes com AH/SD a terem dificuldades de desenvolvimento (Mudrak, 2011; Yazdani \& Daryei, 2016). Já o uso de práticas baseadas em afeto e com limites claros apresentam melhores resultados em termos de desenvolvimento psicossocial (Rudasill, Adelson, Callahan, Houlihan, \& Keizer, 2013).

Para Peterson (2015), famílias de indivíduos talentosos tendem a apresentar características comuns, tais como padrões elevados de desempenho e perfeccionismo e foco em conquistas e sucesso por parte dos pais, o que pode estar associado a estilos educativos mais autoritários (Neumeister \& Finch, 2006). Já Yazdani e Daryei (2016) identificaram os pais de adolescentes superdotados como mais autoritativos quando comparados aos demais, proporcionando melhor autoconceito e diminuição dos níveis de depressão, ansiedade e estresse desses jovens. Morawska e Sanders (2008), que realizaram um estudo com 211 pais e seus filhos, também indicaram que, apesar de as crianças superdotadas não terem mais problemas do que outras, aquelas que apresentaram sintomas e dificuldades relacionais com os pares tinham, na qualidade da relação entre pais e filhos, um fator de proteção. Para Hermann e Lawrence (2012), as famílias de indivíduos com AH/SD são frequentemente estáveis, coesas e responsivas às necessidades da criança, o que pode prevenir o surgimento de problemas associados à pressão para conquistas.

Considerando a escassez de estudos que contemplem as famílias de indivíduos com AH/SD (Renati, Bonfiglio, \& Pfeiffer, 2017) e as incongruências apresentadas, destaca-se a necessidade de pesquisas que continuem investigando fatores relacionados ao sofrimento psíquico de adolescentes com AH/SD com vistas a auxiliar profissionais de saúde e educação a elaborar estratégias de intervenção que proporcionem melhores condições de desenvolvimento a eles e maior suporte a suas famílias (Chung, 2016; Cross \& Cross, 2015; Kennedy \& Farley, 2018; Peterson, 2015). Portanto, este estudo teve como objetivo investigar a associação entre as práticas educativas parentais e os problemas emocionais de comportamento em adolescentes com AH/SD intelectivas. Especificamente, buscou-se identificar as práticas educativas que os adolescentes percebiam como mais utilizadas pelos seus pais, bem como os indicadores de problemas emocionais e de comportamento. Ademais, objetivou-se verificar o poder preditivo das variáveis sociodemográficas e das práticas educativas parentais percebidas em relação a seus problemas emocionais e de comportamento e investigaram-se as diferenças entre os sexos, considerando os dados examinados.

\section{Método}

\section{Delineamento}

Trata-se de um estudo com delineamento observacional e explicativo de corte transversal e abordagem quantitativa (Creswell, 2010). Considerando que as $\mathrm{AH} / \mathrm{SD}$ podem ser observadas em distintas áreas, teve-se como foco de análise as altas habilidades intelectivas identificadas por teste psicométrico, como sugere Virgolim (1997), considerando sua confiabilidade e a dificuldade de operacionalização de uma avaliação global e sistemática das AH/SD (Nakano, Campos, \& Santos, 2016). Tal entendimento está de acordo com a definição adotada no Brasil, já referida na introdução, que indica que indivíduos com AH/SD apresentam potencial elevado e grande desenvolvimento em diferentes áreas do conhecimento, de forma isolada ou combinada (Ministério da Educação, 2009). 


\section{Participantes}

Participaram do estudo 14 adolescentes, dentre os quais sete deles foram acessados por conveniência em uma escola pública do município de Porto Alegre, e os demais por indicação, sendo dois contatados por meio eletrônico (ambos já haviam passado por avaliação de inteligência) e cinco avaliados presencialmente em consultório psicológico. A escola pública escolhida foi indicada pela Secretaria da Educação do Estado do Rio Grande do Sul (Seduc) por ter Atendimento Educacional Especializado (AEE) a alunos com AH/SD, que já haviam sido previamente identificados pelos critérios escolares para compor tal grupo.

A amostra foi composta por 10 adolescentes do sexo masculino $(71,4 \%)$ e quatro do sexo feminino $(28,6 \%)$ e a idade variou de 12 a 16 anos $(\mathrm{M}=13,3$; $\mathrm{DP}=1,4)$. As mães apresentavam idade média de 41,1 anos (DP 8,9), e os pais idade média de 47,6 anos (DP $=10,1)$. Sobre o nível de escolaridade, prevaleceu o ensino superior (33,3\% das mães, e $50 \%$ dos pais), e a maior parte deles tinha trabalho remunerado no momento da investigação (64,3\% das mães e 92,9\% dos pais). As famílias dos adolescentes que participaram da pesquisa tinham renda de dois a 10 salários-mínimos, indicando que pertenciam às classes sociais B e C, conforme indicadores sociais do IBGE (Instituto Brasileiro de Geografia e Estatísticas, 2018).

Todos os adolescentes considerados apresentavam quociente de inteligência (QI) acima da média, ou seja, acima de 100 pontos (QI Verbal: $\mathrm{M}=143,64$; $\mathrm{DP}=13,71 ;$ QI Execução: $\mathrm{M}=127,14 ; \mathrm{DP}=17,37$; QI Total: $\mathrm{M}=137,86 ; \mathrm{DP}=14,89$ ), na Escala de Inteligência Wechsler Abreviada, também chamada de WASI (Trentini, Yates, \& Heck, 2014). Esse instrumento fornece informações sobre o QI total, de execução e verbal a partir de quatro subtestes (vocabulário, cubos, semelhanças e raciocínio matricial) em um curto espaço de tempo.

\section{Instrumentos}

Questionário de Dados Sociodemográficos adaptado de Núcleo de Infância e Família (2008): obtém informações sobre os dados sociodemográficos do adolescente e sua família (idade, escolaridade e sexo, além da configuração familiar, situação conjugal, acadêmica e profissional dos pais).

Escalas de Práticas Parentais (EPP) (Teixeira, Oliveira, \& Wottrich, 2006): avalia as dimensões das práticas educativas maternas e paternas (controle punitivo, supervisão do comportamento, cobrança de responsabilidade, intrusividade, apoio emocional e incentivo à autonomia) a partir de 27 itens respondidos por meio de uma escala de resposta do tipo Likert de cinco pontos (sendo 1 equivalente a quase nunca ou bem pouco e 5 a quase sempre ou bastante). Suas propriedades psicométricas são consideradas satisfatórias (consistência interna das escalas de pais e mães variaram de 0,65 a 0,90) e, neste estudo, o alpha de Cronbach estimado pelo método split half para os componentes paternos oscilou de 0,81 (intrusividade) a 0,96 (apoio emocional), apontando para consistências classificadas de altas $(0,75<\alpha \leq 0,90)$ a muito altas $(\alpha>0,90)$. Sobre os componentes maternos, foram encontradas confiabilidades classificadas como muito altas em controle punitivo $(\alpha=0,94)$ e incentivo à autonomia $(\alpha=0,95)$, alta em apoio emocional $(\alpha=0,86)$ e moderada na dimensão intrusividade $(\alpha=0,74)$. Já a supervisão de comportamento e a cobrança de responsabilidade alcançaram coeficientes de 0,61 ( $p<0,03)$ e $0,46(p<0,04)$. Todos os indicadores são considerados aceitáveis, dada a limitação do tamanho amostral deste estudo.

Inventário de Comportamentos Autorreferidos para jovens de 11 a 18 anos - Youth Self Report (YSR) (Achenbach \& Rescorla, 2001, 2004): avalia a saúde mental de adolescentes por meio de indicadores autorreferidos de competência e problemas de comportamento e/ou psicológicos. Neste estudo, utilizou-se apenas o segundo nível do instrumento, que é formado por 105 itens que examinam indicadores de problemas emocionais e de comportamento. Os dados fornecem um perfil de oito escalas de síndromes, que, quando associadas, derivam um total de problemas internalizantes e externalizantes. Os problemas internalizantes são compostos pelas escalas de ansiedade/ depressão, isolamento/depressão e queixas somáticas; já os problemas externalizantes englobam as escalas de comportamento de quebra de regras e comportamento agressivo. Por último, existem os fatores gerais que abarcam as escalas de problemas sociais, problemas de pensamento e problemas de atenção, além de 14 itens que se referem a comportamentos socialmente desejáveis (escala de aspectos positivos). O instrumento ainda contempla escalas orientadas pelo DSM, mas estas não foram analisadas neste estudo. Em relação às propriedades psicométricas, o YSR se encontra em processo de validação para a população brasileira. Porém, já foram apontados 
índices de consistência interna adequados, como o índice RMSEA de 0,03, indicando bom ajustamento (Rocha, 2012). Além disso, Bordin et al. (2013) também revelaram que a confiabilidade média de teste-reteste foi de 0,82 , e a consistência interna das escalas de problemas, medida por alfa de Cronbach, variou de 0,67 a 0,95. Em relação à consistência interna do YSR neste estudo, ela foi classificada como baixa no fator quebra de regras $(\alpha=0,59)$, e moderada nas escalas problemas sociais $(\alpha=0,73)$ e isolamento/depressão $(\alpha=0,64)$. Os demais coeficientes de confiabilidade concentraram-se na classificação alta $(0,75<\alpha \leq 0,90)$.

\section{Procedimentos éticos e de coleta de dados}

Este estudo foi aprovado pelo Comitê de Ética em Pesquisa da Universidade do Vale do Rio dos Sinos (CAAE: 66512817.0.0000.5344). Tanto os adolescentes quanto seus pais ou representantes legais foram informados a respeito dos objetivos e procedimentos metodológicos e ficaram livres para dar seu consentimento para a realização da pesquisa.

A coleta de dados ocorreu em diferentes locais. $\mathrm{Na}$ escola, após aprovação da Coordenadoria de Educação do Estado (1 ${ }^{\text {a }} \mathrm{CRE}$ ) e da direção, foi realizado contato com os pais dos adolescentes identificados como tendo AH/SD (por meio da indicação pela professora da sala de AEE) para esclarecimento sobre a pesquisa e obtenção do Termo de Consentimento Livre e Esclarecido (TCLE). Em seguida, foi agendado um encontro na escola, quando os adolescentes assinaram o Termo de Assentimento (TA) e responderam aos instrumentos, inclusive ao WASI, para confirmação do potencial cognitivo.

Nas coletas realizadas por meio eletrônico, inicialmente contatava-se, via e-mail, os pais dos adolescentes para explicar a pesquisa e obter informações sobre o diagnóstico de AH/SD. Após confirmação da AH/SD intelectiva por meio dos resultados do WASI, era encaminhado, por e-mail, os TCLE para os pais e o TA para o adolescente, bem como o questionário sociodemográfico para preenchimento e devolução pela mesma via. Em seguida, era agendado um horário com o adolescente para a aplicação dos instrumentos EPP e YSR, via Skype. Nesse momento, apresentava-se o instrumento ao adolescente, cuja aplicação era feita na forma de entrevista.

Em relação às coletas que foram realizadas em consultório psicológico, novamente foi feito contato telefônico inicial com os pais, e, ao demonstrarem interesse e apontarem que seu filho(a) tinha indicação de AH/SD, era-lhes explicada a pesquisa. Posteriormente, em encontro presencial, era entregue e assinado o TCLE. Então agendava-se encontro com os adolescentes, quando assinavam o TA e eram aplicados os instrumentos de forma individual. O tempo de aplicação dos instrumentos foi de aproximadamente 60 minutos. A primeira autora sempre esteve presente, realizando a leitura das instruções de preenchimento e à disposição para sanar dúvidas e oferecer esclarecimentos durante todo o processo.

\section{Procedimentos de análise de dados}

As respostas ao Questionário de Dados Sociodemográficos foram analisadas por meio de estatística descritiva para caracterização da amostra. Foram utilizadas as distribuições, absoluta (n) e relativa (\%), bem como as medidas de tendência central e dispersão (média, mediana e desvio-padrão) com o estudo de normalidade da distribuição dos dados pelo teste de Kolmorogov-Smirnov (correção de Lillifors), que indicou distribuição normal dos dados.

OsinstrumentosWASI,EPPeYSRforamanalisados de acordo com seus manuais. OYSR, especificamente, foi analisado a partir do ASEBA-PC, software central do Sistema de Avaliação Empiricamente Baseado de Achenbach (Achenbach System of Empirically Based Assessment-ASEBA). As associações entre as variáveis foram analisadas por meio da Correlação de Pearson e o Teste $t$ de Student para amostras independentes.

Por fim, foi empregada a técnica de Análise de Regressão Linear Múltipla (método backward stepwise). Foram verificados os pressupostos de normalidade e homogeneidade dos erros e de sua independência (estatística de Durbin Watson), de acordo com Field (2009) e Marôco (2011). Para o diagnóstico de multicolinearidade, foram usados os valores variance inflation factor (VIF). Todos os dados foram analisados por meio do software de análise estatística Statistical Package for the Social Sciences (SPSS), versão 20.0, considerando o nível de significância $p<0,05$.

\section{Resultados}

Inicialmente, avaliou-se a percepção que os adolescentes tinham das práticas educativas parentais. Os resultados indicaram que as mães apresentaram índices mais elevados do que os pais nas dimensões cobrança de responsabilidade (Mãe: $\mathrm{M}=4,42$, $\mathrm{DP}=0,66$; Pai: $\mathrm{M}=3,87, \mathrm{DP}=1,21$ ), apoio emocional 
(Mãe: $\mathrm{M}=4,15, \mathrm{DP}=0,83$; Pai: $\mathrm{M}=3,82, \mathrm{DP}=1,24$ ) e incentivo à autonomia (Mãe: $\mathrm{M}=4,14, \mathrm{DP}=1,12$; Pai: $\mathrm{M}=3,50, \mathrm{DP}=1,26$ ). A menor média foi observada na dimensão intrusividade materna $(\mathrm{M}=2,96$, $\mathrm{DP}=1,08)$ e paterna $(\mathrm{M}=2,53, \mathrm{DP}=1,09)$. Os dados são apresentados na Tabela 1.
Quanto aos indicadores de problemas emocionais e de comportamento, destacaram-se com maiores médias os problemas internalizantes $(50 \%)$ e suas escalas: ansiedade/depressão, isolamento/depressão, problemas sociais e problemas de pensamento. Na Tabela 2 são apresentados os dados.

Tabela 1

Média e desvio-padrão das práticas educativas maternas e paternas.

\begin{tabular}{ccccc}
\hline Dimensões & \multicolumn{2}{c}{ Mãe } & Pai \\
\cline { 2 - 5 } & Média & DP & Média & DP \\
\hline Apoio emocional & 4,15 & 0,83 & 3,82 & 1,24 \\
Controle punitivo & 3,57 & 1,42 & 3,14 & 1,49 \\
Incentivo autonomia & 4,14 & 1,12 & 3,50 & 1,26 \\
Intrusividade & 2,96 & 1,08 & 2,53 & 1,09 \\
Supervisão comportamento & 4,48 & 0,64 & 4,14 & 1,09 \\
Cobrança de responsabilidade & 4,42 & 0,66 & 3,87 & 1,21 \\
\hline
\end{tabular}

\section{Tabela 2}

Média, desvio-padrão e distribuição absoluta e relativa para os indicadores de problemas emocionais e de comportamento.

\begin{tabular}{ccccccccc}
\hline \multirow{2}{*}{ Escalas } & \multicolumn{9}{c}{ Normal } & \multicolumn{1}{c}{ Limítrofe } & \multicolumn{2}{c}{ Clínico } \\
\cline { 2 - 10 } & \multicolumn{9}{c}{ Média } & DP & $\mathbf{n}$ & $\mathbf{\%}$ & $\mathbf{n}$ & $\mathbf{\%}$ & $\mathbf{n}$ & $\mathbf{\%}$ \\
\cline { 2 - 10 } & 64,14 & 10,71 & 7 & 50,0 & 3 & 21,4 & 4 & 28,6 \\
Ansiedade e depressão & 61,43 & 10,29 & 10 & 71,4 & 3 & 21,4 & 1 & 7,1 \\
Isolamento e depressão & 58,50 & 11,03 & 11 & 78,6 & 1 & 7,1 & 2 & 14,3 \\
Queixas somáticas & 60,64 & 10,09 & 9 & 64,3 & 2 & 14,3 & 3 & 21,4 \\
Problemas sociais & 60,50 & 12,17 & 10 & 71,4 & 1 & 7,1 & 3 & 21,4 \\
Problemas de pensamento & 56,64 & 8,95 & 12 & 85,7 & & & 2 & 14,3 \\
Problemas de atenção & 53,71 & 4,87 & & & & & & \\
Quebra de regras & 56,14 & 6,87 & 12 & 85,7 & 1 & 7,1 & 1 & 7,1 \\
Comportamento agressivo & 62,50 & 12,52 & 6 & 42,9 & 1 & 7,1 & 7 & 50,0 \\
Problemas internalizantes & 51,89 & 9,35 & 10 & 71,4 & 3 & 21,4 & 1 & 7,1 \\
Problemas externalizantes & 58,71 & 11,84 & 8 & 57,1 & 2 & 14,3 & 4 & 28,6 \\
Total de problemas & 47,93 & 12,30 & 10 & 71,4 & 4 & 28,6 & & \\
Aspectos positivos & & & & & & &
\end{tabular}

As variáveis consideradas também foram comparadas quanto ao sexo. Verificou-se que, em relação às práticas educativas, de modo geral as meninas apresentaram pontuações médias mais elevadas, exceto no componente controle punitivo mãe, mas as diferenças não se mostraram significativas.
Sobre os indicadores emocionais e de comportamento, os meninos apresentaram mais indicadores de problemas internalizantes $(\mathrm{p}<0,03$; Meninas: $\mathrm{M}=51,25, \mathrm{DP}=4,34$; Meninos: $\mathrm{M}=67,00, \mathrm{DP}=11,89$ ), problemas externalizantes $(\mathrm{p}<0,03$; Meninas: $\mathrm{M}=43,50, \mathrm{DP}=4,93$; Meninos: $\mathrm{M}=55,10, \mathrm{DP}=8,69$ ) 
e total de problemas ( $\mathrm{p}<0,02$; Meninas: $\mathrm{M}=47,75$, $\mathrm{DP}=5,90$; Meninos: $\mathrm{M}=63,10, \mathrm{DP}=10,77$ ), superando entre $26 \%$ e $32 \%$ a média das meninas. De modo semelhante, os meninos apresentaram médias mais altas nas escalas isolamento/depressão ( $\mathrm{p}<0,02$; Meninas: $\mathrm{M}=52,00, \mathrm{DP}=2,00$; Meninos: $\mathrm{M}=65,20, \mathrm{DP}=9,81)$ e problemas sociais $(\mathrm{p}<0,002$; Meninas: $\mathrm{M}=51,25$; $\mathrm{DP}=0,95$; Meninos: $\mathrm{M}=64,40$, $\mathrm{DP}=9,59$ ). Com menor magnitude, mas ainda significativa, houve diferença nas escalas: queixas somáticas ( $\mathrm{p}=0,04$; Meninas: $\mathrm{M}=51,75, \mathrm{DP}=1,0$; Meninos: $\mathrm{M}=61,20, \mathrm{DP}=12,10)$ e comportamento agressivo ( $p=0,003$; Meninas: $M=50,00, D P=0,00$; Meninos: $\mathrm{M}=58,60, \mathrm{DP}=6,68$ ).

Em relação à associação entre as práticas educativas maternas e os indicadores de problemas emocionais e de comportamento, os resultados da análise de correlação apontaram que o componente apoio emocional se associou de forma significativa, negativa e classificada como moderada, com queixas somáticas $(r=-0,69 ; \mathrm{p}<0,01)$, problemas de pensamento $(\mathrm{r}=-0,59 ; \mathrm{p}=0,02)$, problemas somáticos $(r=-0,59 ; \mathrm{p}=0,03)$ e problemas obsessivos compulsivos $(r=-0,62 ; \mathrm{p}=0,02)$. Já o componente controle punitivo apontou correlações significativas e negativas classificadas como fortes com a idade mãe $(r=-0,73$; $\mathrm{p}<0,003)$ e a idade pai $(\mathrm{r}=-0,81 ; \mathrm{p}=0,003)$. Ainda houve correlação significativa, mas negativa e moderada, entre controle punitivo e aspectos positivos $(r=-0,55$; $\mathrm{p}=0,04)$. O componente incentivo à autonomia teve correlações classificadas como moderadas com problemas de atenção $(r=-0,57 ; p=0,03)$, problemas internalizantes $(r=-0,56 ; p=0,04)$, problemas afetivos $(\mathrm{r}=-0,55 ; \mathrm{p}=0,005)$ e problemas de ansiedade $(r=-0,63 ; p=0,01)$. No que se refere ao pai, apenas o incentivo à autonomia se correlacionou de forma fraca e negativa com sua idade $(r=-0,60 ; p=0,05)$.

Por fim, verificou-se o poder preditivo das práticas educativas parentais percebidas em relação aos seus problemas emocionais e de comportamento. Foi estimado um modelo de regressão, considerando os resultados apresentados pela análise de correlação com nível mínimo de significância inferior ou igual a $20 \%(\mathrm{p} \leq 0,20)$ para compor modelos múltiplos iniciais (Hair Jr., Black, Babin, Anderson, \& Tatham, 2009). Para determinação do modelo múltiplo, adotou-se o método de seleção stepwise backward, de forma que eles foram sendo ajustados, removendo-se uma a uma as variáveis independentes (práticas educativas) que não se mostraram associadas à variável dependente (indicadores de problemas emocionais e de comportamento). O percentual da variância explicada pelo modelo foi estimado por meio do coeficiente de determinação ajustado ( $\mathrm{R}^{2}$ ajustado).

Para responder às variações dos indicadores de problemas internalizantes, o modelo inicial foi composto pelas dimensões apoio emocional da mãe $(\mathrm{r}=-0,42 ; \mathrm{p}=0,13)$, incentivo à autonomia pela mãe $(r=-0,56 ; p<=0,04)$, incentivo à autonomia pelo pai $(\mathrm{r}=-0,36 ; \mathrm{p}=0,19)$ e intrusividade da mãe $(\mathrm{r}=0,36$; $\mathrm{p}=0,20)$. De acordo com os resultados obtidos, o modelo final foi elencado em quatro etapas $\left(\mathrm{F}_{1 ; 12}=5,4\right.$; $\left.\mathrm{p}=0,03 ; \mathrm{R}_{\text {modelo }}=0,25\right)$ e mostrou-se significativo para o incentivo à autonomia pela mãe $\left(b_{p}=-0,56\right.$; $p=0,03 ; R_{p}^{2}=0,31$ ). Dessa forma, $25,3 \%$ das variações observadas nos indicadores de problemas internalizantes foram explicadas por essa variável.

Na determinação do modelo de regressão múltipla para responder pela variação dos indicadores de problemas externalizantes, foi elencado para o modelo apenas o apoio emocional da mãe $(r=-0,42 ; p=0,14)$. Em relação à estimativa obtida para o modelo final $\left(\mathrm{F}_{1-12}=2,5 ; \mathrm{p}=0,13 ; \mathrm{R}_{\text {modelo }}{ }=0,10\right)$, os resultados apontaram que $10,7 \%$ das variações observadas nas pontuações dos problemas externalizantes mostraram-se relacionadas ao apoio emocional materno $\left(b_{p}=-0,41\right.$; $\mathrm{p}=0,13 ; \mathrm{R}_{\mathrm{p}}^{2}=0,17$ ).

Na estimativa do modelo para explicar os indicadores totais de problemas, as variáveis independentes que compuseram o modelo inicial foram: a) apoio emocional da mãe $(r=-0,46 ; p=0,10) ; b)$ incentivo à autonomia pela mãe $(r=-0,49 ; p=0,08)$; e c) incentivo à autonomia pelo pai $(r=-0,36 ; p=0,20)$. A seleção do modelo final ocorreu em três etapas $\left(\mathrm{F}_{1-12}=3,7\right.$; $\mathrm{p}=0,07 ; \mathrm{R}_{\text {modelo }}=0,17$ ), permanecendo como significativo o incentivo à autonomia pela mãe $\left(\mathrm{b}_{\mathrm{p}}=-0,49\right.$; $\left.\mathrm{p}=0,07 ; \mathrm{R}_{\mathrm{p}}^{2}=0,24\right)$. Pelas estimativas obtidas, verificou-se que $17,4 \%$ das variações observadas na escala total de problemas conseguem ser explicadas pelo incentivo à autonomia pela mãe.

No que se refere ao modelo de regressão para responder pela variação observada nos indicadores de aspectos positivos, foram elencadas, para o modelo inicial, o controle punitivo da mãe $(r=-0,55 ; p=0,04)$ e do pai $(r=-0,49 ; p=0,07)$, intrusividade da mãe $(\mathrm{r}=-0,46 ; \mathrm{p}=0,10)$ e cobrança de responsabilidade pela mãe $(p=-0,55 ; p=0,04)$. Sobre a estimativa para o modelo final, elencado em quatro etapas $\left(\mathrm{F}_{1-12}=5,30\right.$; 
$\left.\mathrm{p}=0,04 ; \mathrm{R}_{\text {modelo }}=0,25\right)$, os resultados indicaram que $24,9 \%$ das variações observadas para os indicadores de aspectos positivos podem ser explicadas pelo controle punitivo da mãe $\left(b_{p}=-0,55 ; p=0,04 ; R_{p}^{2}=0,31\right)$.

\section{Discussão}

Tendo em vista os objetivos do estudo e os dados obtidos, constatou-se que os adolescentes participantes indicaram que suas mães demonstravam mais cobrança de responsabilidade, apoio emocional e incentivo à autonomia do que seus pais. De fato, na população geral, as mães têm sido indicadas por alguns estudos como mais atuantes do que os pais em relação à educação dos filhos (Costa, Teixeira, \& Gomes, 2000; Freitas \& Piccinini, 2010). Especificamente no contexto das AH/SD, as mães costumam se envolver mais na educação, estimulando o desenvolvimento socioemocional dos adolescentes (Alvino, 1995; Hidalgo, 2016).

Além disso, identificou-se que o fator idade do pai esteve associado a algumas diferenças nas práticas educativas. Pais mais jovens, como os deste estudo, tendem a exercer mais controle (Valcke, Bonte, Wever, \& Rots, 2010) em relação aos mais velhos (Pilarinos \& Solomon, 2017), o que pode estar associado ao menor incentivo à autonomia de seus filhos, tão importante na adolescência, independentemente do nível intelectual. No estudo de Freeman (2014) com meninos e meninas superdotados, constatou-se que eles percebiam o uso de práticas educativas mais restritivas por parte de seus pais, em razão de comumente serem mais jovens que seus colegas, especialmente quando aceleravam os estudos.

Já no que diz respeito à presença de problemas emocionais e de comportamento, os adolescentes com AH/SD que participaram deste estudo apresentaram mais indicadores de problemas internalizantes, assim como nas escalas de ansiedade/depressão, isolamento/depressão, problemas sociais e de pensamento. Portanto, corrobora-se a literatura que sugere haver um descompasso entre alta capacidade cognitiva e maturidade afetiva (Ourofino \& Fleith, 2011). A maior curiosidade e facilidade para adquirir conhecimentos que o superdotado tem pode gerar um desgaste mental devido à dessincronia entre sua idade e o desenvolvimento emocional, podendo decorrer disso sentimentos de desânimo e frustrações, que também foram indicados em outros estudos com a mesma população, o que pode levar a quadros de ansiedade e depressão
(Cross \& Cross, 2015; Eren et al., 2018; Neihart, 2002; Probst \& Piechowski, 2012; Silverman, 2002).

Quanto à associação entre as práticas educativas parentais e os problemas emocionais e de comportamento em adolescentes com AH/SD, os resultados deste estudo revelaram que o baixo apoio emocional materno se relacionou com mais queixas somáticas e problemas de pensamento. Ainda, aqueles que percebiam o menor incentivo à autonomia por parte da mãe apresentaram pontuações mais elevadas nos problemas de atenção, problemas internalizantes e problemas de ansiedade. Para Freeman (2014) e Fugatea e Gentryb (2016), o comportamento dos pais pode diminuir a autoconfiança dos filhos e aumentar os sentimentos de insatisfação. É plausível supor que o menor incentivo à autonomia reflita em um estilo mais autoritário por parte dessas mães, por não estarem estimulando o desenvolvimento e a independência de seus filhos. Nesse sentido, mesmo tendo capacidade intelectual elevada, não haveria estímulo ao desenvolvimento emocional, o que pode explicar, ao menos em parte, a maior incidência de problemas internalizantes neste estudo.

Apoiando o exposto, os resultados também indicaram quehouve correlação negativa emoderada entre o controle punitivo exercido pelos pais e os aspectos positivos dos adolescentes. Para Alvino (1995), desde a infância os pais são importante influência para o desenvolvimento de superdotados, destacando que, entre as práticas educativas, conversar sobre sentimentos e emoções, elogiar e interagir com os filhos ajuda a aumentar seu autoconceito. Nessa mesma direção, Olszewski-Kubilius, Lee e Thomson (2014) sinalizaram que ambientes familiares mais afetivos promovem o desenvolvimento de habilidades e competências em jovens superdotados. Por outro lado, Ayğari \& Gündoğdu (2017) sinalizaram que os estilos parentais permissivos/negligentes e autoritários impactam negativamente a autopercepção de jovens com AH/SD.

Quando os adolescentes foram comparados entre os sexos, evidenciou-se que os meninos apresentaram médias mais altas em relação ao total de problemas internalizantes, externalizantes e total de problemas. Eles constituíam a maioria da amostra e foram identificados como tendo mais dificuldades emocionais e comportamentais do que as meninas, que, por sua vez, apresentaram mais indicadores de saúde mental. Tais dados são sustentados por Ogurlu et al. (2016), 
Eren et al. (2018) e Tentes e Fleith (2014), que afirmaram a existência de diferenças entre meninos e meninas superdotados, sinalizando que as meninas têm uma avaliação mais positiva de si mesmas. Além disso, segundo Alencar (2007a), os meninos costumam apresentar maior desigualdade entre a capacidade mental e comportamental, o que contribui para que tenham mais dificuldades de relacionamento social, gerando empobrecimento das habilidades sociais e da autoimagem, além de incremento dos sentimentos de isolamento e solidão.

Por fim, verificou-se o poder preditivo das práticas educativas parentais percebidas em relação aos problemas emocionais e de comportamento dos adolescentes com AH/SD. Os resultados revelaram que $25,3 \%$ das variações observadas para os indicadores de problemas internalizantes eram explicadas pelo baixo incentivo à autonomia por parte da mãe, e $10,7 \%$ das variações observadas para os indicadores de problemas externalizantes eram explicados pela baixa percepção de apoio emocional também da mãe. Já o total de problemas foi explicado em $17,4 \%$ dos casos pela menor percepção de incentivo à autonomia pela mãe, enquanto $24,9 \%$ das variações observadas no desenvolvimento dos aspectos positivos foram atribuídas à percepção de menor controle punitivo materno. Assim, constata-se que as dificuldades emocionais e comportamentais identificadas entre os adolescentes com AH/SD da amostra foram mais bem explicadas por práticas educativas maternas. Conclui-se, portanto, que, quando as mães são mais atentas e afetivas, tendem a estabelecer regras e limites claros, apresentando expectativas admissíveis, que repercutirão em melhores resultados na condução da educação de seus filhos com AH/SD (Olszewski-Kubilius et al., 2014; Rudasill, et al., 2013). A não associação com as práticas educativas paternas neste estudo pode ser explicada pelo fato de os pais de superdotados tenderem a demonstrar maior ansiedade e terem mais dificuldade em lidar com questões emocionais dos filhos quando comparados às mães, o que pode fazer com que participem menos de sua educação (Hidalgo, 2016).

Em conjunto, os dados indicam que os adolescentes que participaram deste estudo, especialmente os meninos, que constituíram a maioria da amostra, apresentavam indicadores de problemas emocionais e de comportamento caracterizados por sintomas tanto externalizantes como internalizantes.
As meninas, por sua vez, exibiram mais aspectos positivos, o que poderia indicar que sua saúde mental estava mais preservada.

Evidenciou-se a importância de avaliar como esses adolescentes se sentem, se comportam e como percebem a interação com seus pais, particularmente a forma como as mães os educam. Tais resultados confirmam a importância dos pais no desenvolvimento de filhos com AH/SD e em prepará-los para o desempenho de suas funções por meio de informações e programas psicoeducativos que abordem as necessidades dos filhos, objetivando que assumam uma atitude de apoio (Ayğari \& Gündoğdu, 2017) e estímulo à autonomia, considerando que se trata de práticas fundamentais para o desenvolvimento adolescente.

\section{Considerações finais}

Embora a literatura seja ampla quanto aos estudos que tratam das AH/SD, em especial no que tange ao seu desenvolvimento comportamental e emocional (Alencar, 2015), ainda há pouca ênfase sobre aspectos familiares. Diante disso, acredita-se que este estudo tenha colaborado com o conhecimento na área, assinalando a contribuição das práticas educativas parentais e o quanto explicam os indicadores de problemas emocionais e de comportamento em adolescentes com AH/SD intelectivas. Destacou-se a importância da mãe no contexto familiar (Costa et al., 2000) e o quanto as práticas educativas utilizadas por ela podem afetar o desenvolvimento de filhos com AH/SD (Alvino, 1995). Também se concluiu que a superdotação não pode ser considerada necessariamente como um fator protetivo (Jones, 2013; Richards et al., 2003) contra problemas emocionais e comportamentais (Borges del Rosal, HernándezJorge, \& Rodríguez-Naveiras, 2011; Francis, Hawes, \& Abbott, 2016), na expectativa de que as altas habilidades cognitivas também impliquem melhor ajuste psicossocial. Destaca-se que uma especial atenção deve ser dada aos meninos, uma vez que estes parecem ficar mais vulneráveis aos problemas emocionais e de comportamento, especialmente quando suas mães tendem a ser menos próximas afetivamente e não conseguem incentivar sua autonomia, questão fundamental na fase adolescente.

Por fim, cabe ressaltar as limitações deste estudo, a começar pela reduzida amostra resultante da dificuldade de encontrar participantes, especialmente devido à restrita estrutura escolar estadual que ainda 
não está capacitada para identificar alunos com AH/SD. Tal questão limita o poder das análises estatísticas realizadas. No mesmo sentido, a diferença no quantitativo de meninos e meninas também pode ser considerada uma limitação. Contudo, essa questão parece seguir uma tendência, uma vez que, em meta-análise realizada por Petersen (2013), identificou-se que os meninos tendem a ser mais frequentemente identificados quando são utilizadas medidas de QI ou testes padronizados e, consequentemente, participam mais de estudos e programas direcionados a AH/SD. Ademais, os jovens que participaram da pesquisa podem ter sido indicados, seja pela escola, seja por seus pais, justamente por já apresentarem algum tipo de problema emocional ou comportamental previamente verificado, na expectativa de que encontrariam acolhimento a suas demandas, fazendo parte de um estudo na área da psicologia, como de fato ocorreu em alguns casos $(n=2)$.

Destaca-se que este trabalho pretende servir de apoio a futuras pesquisas sobre o tema das AH/SD, possibilitando a fundamentação de programas para o treinamento de habilidades para os adolescentes e seus pais, a serem desenvolvidos por profissionais psicólogos e outros que se dediquem ao estudo do tema. Seus dados também poderão servir de informação a familiares e comunidade escolar, considerando a falta de conhecimento e as muitas dúvidas sobre como interagir com indivíduos que tenham essas características (Renati et al., 2017).

\section{Referências}

Achenbach, T. M., \& Rescorla, L. A. (2001). Manual for the ASEBA school-age forms, \& profiles. University of Vermont.

Achenbach, T. M., \& Rescorla, L. A. (2004). Mental health practitioners' guide for the Achenbach System of Empirically Based Assessment (ASEBA). University of Vermont.

Alencar, E. M. L. S. (2007a). Características sócio-emocionais do superdotado: questões atuais. Psicologia em Estudo, 12(2), 371-378. https://doi.org/10.1590/S1413-73722007000200018

Alencar, E. M. L. S. (2007b). Indivíduos com AH/SD: clarificando conceitos, desfazendo ideias errôneas. In D. S. Fleith (Ed.), A construção de práticas educacionais para alunos com AH/SD: orientação a professores (pp. 15-23). MEC; Seesp.

Alencar, E. M. L. S. (2015). Contribuições de estudos de caso para o avanço do conhecimento sobre superdotação. Psicologia Escolar e Educacional, 19(3), 427-434. https://doi.org/10.1590/2175-3539/2015/0193830

Alvino, J. (1995). Considerations and strategies for parenting the gifted child. The National Research Center on the Gifted and Talented.

Ayğar, B. B., \& Gündoğdu, M. (2017). The relationship between gifted and ungifted students' self-perceptions and their parents' parenting styles: A structural equation model. European Journal of Education Studies, 3(3), 334-350. https:// doi.org/10.5281/zenodo.29295

Bordin, I. A., Rocha, M. M., Paula, C. S., Teixeira, M. C. T. V., Achenbach, T. M., Rescorla, L. A., \& Silvares, E. F. M. (2013). Child behavior checklist (CBCL), youth self-report (YSR) and teacher's report form (TRF): An overview of the development of the original and Brazilian versions. Cadernos de Saúde Pública, 29(1), 13-28. https://doi.org/ $10.1590 /$ S0102-311X2013000100004

Borges del Rosal, A., Hernández-Jorge, C., \& Rodríguez-Naveiras, E. (2011). Evidencias contra el mito de la inadaptación de las personas con altas capacidades intelectuales. Psicothema, 23(3), 362-367.

Chung, E. Y. H. (2016). School counselling for the gifted: Responding to the social-emotional needs of gifted students. In M.-T. Hue (Ed.), School counselling in a Chinese context: Supporting students in need in Hong (pp. 102-113). Routledge.

Chung, D., Yun, K., Kim, J. H., Jang, B., \& Jeong, J. (2011). Different gain/loss sensitivity and social adaptation ability in gifted adolescents during a public goods game. PloS one, 6(2). https://doi.org/10.1371/journal.pone.0017044

Costa, F. T., Teixeira, M. A. P., \& Gomes, W. B. (2000). Responsividade e exigência: Duas escalas para avaliar estilos parentais. Psicologia Reflexão e Crítica, 13(3), 465-473. http://doi.org/10.1590/S0102-79722000000300014

Creswell, J. W. (2010). Projeto de pesquisa: Métodos qualitativo, quantitativo e misto. Artmed.

Cross, J. R., \& Cross, T. L. (2015). Clinical and mental health issues in counseling the gifted individual. Journal of Counseling \& Development, 93(2), 163-172. https://doi.org/10.1002/j.1556-6676.2015.00192.x 
Psicologia: Ciência e Profissão 2021 v. 41(n. spe 3), e203883, 1-15.

Dalosto, M. M., \& Alencar, E. M. L. S. (2013). Manifestações e prevalência de bullying entre alunos com AH/SD. Revista Brasileira de Educação Especial, 19(3), 363-378. http://doi.org/10.1590/S1413-65382013000300005

Eren, F., Çete, A. Ö., Avcil, S., \& Baykara, B. (2018). Emotional and behavioral characteristics of gifted children and their families. Archives of Neuropsychiatry, 55(2), 105. https://doi.org/10.5152/npa.2017.12731

Field, A. (2009). Discovering statistics using SPSS (3a ed). Sage Publications.

França-Freitas, M. L. P., Del Prette, A., \& Del Prette, Z. A. P. (2014). Social skills of gifted and talented children. Estudos de Psicologia, 19(4), 288-295. http://doi.org/10.1590/S1413-294X2014000400006

Francis, R., Hawes, D. J., \&Abbott, M. (2016). Intellectual giftedness and psychopathology in children and adolescents: A systematic literature review. Exceptional Children, 82(3), 279-302. https://doi.org/10.1177/0014402915598779

Freeman, J. (2014). Um estudo comparativo de 35 anos com crianças identificadas como superdotadas, não identificadas como superdotadas e com habilidades médias. Revista Educação Especial, 27(50), 563-581. https://doi.org/ $10.5902 / 1984686 \times 14677$

Freitas, A. P. C. O., \& Piccinini, C. A. (2010). Práticas educativas parentais em relação ao filho único e ao primogênito. Estudos de Psicologia, 27(4), 515-528. http://doi.org/10.1590/S0103-166X2010000400009

Fugatea, C. M., \& Gentryb, M. (2016). Understanding adolescent gifted girls with ADHD: Motivated and achieving. High Ability Studies, 27(1), 83-109. https:// doi.org/10.1080/13598139.2015.1098522

Gallucci, N. T., Middleton, G., \& Kline, A. (1999). Intellectually superior children and behavioral problems and competence. Roeper Review, 22(1), 18-21. https:// doi.org/10.1080/02783199909553992

Garland, A. F, \& Zigler, E. (1999). Emotional and behavioral problems among highly intellectually gifted youth. Roeper Review, 22(1), 41-44. https://doi.org/10.1080/02783199909553996

Hair Jr., J. F., Black, W. C., Babin, B. J., Anderson, R. E., \& Tatham R. L. (2009). Análise multivariada de dados (6a ed). Bookman.

Hakin, C. (2016). Superdotação e dupla excepcionalidade. Juruá.

Hermann, K. M., \& Lawrence, C. (2012). Family relationships. In T. L. Cross, \& J. R. Cross (Eds.), Handbook for counselors serving students with gifts and talents (pp. 393-407). Prufrock.

Hidalgo, M. F. (2016). Parenting the gifted and talented child: A qualitative inquiry of the perceptions of mothers regarding their unique experiences in raising gifted and talented children [Tese de Doutorado, Louisiana State University]. Louisiana State University Digital Commons. https://digitalcommons.lsu.edu/cgi/viewcontent.cgi? article $=5275 \&$ context=gradschool_dissertations

Instituto Brasileiro de Geografia e Estatística. (2018). Síntese de indicadores sociais. https://www.ibge.gov.br/ estatisticas/sociais/populacao/9221-sintese-de-indicadores-sociais.html?edicao=23289\&t=resultados

Instituto Nacional de Estudos e Pesquisas Educacionais Anísio Teixeira. (2014). Sinopse e estatística da educação básica 2018. http://portal.inep.gov.br/web/guest/sinopses-estatisticas-da-educacao-basica

Jones, T. W. (2013). Equally cursed and blessed: Do gifted and talented children experience poorer mental health and psychological well-being? Educational \& Child Psychology, 30(2), 44-66.

Kennedy, K., \&Farley, J. (2018). Counseling gifted students: School-based considerations and strategies. International Electronic Journal of Elementary Education, 10(3), 361-367. https://doi.org/10.26822/iejee.2018336194

Lei n. 13.234. (2015). Altera a Lei no 9.394, de 20 de dezembro de 1996. Diário Oficial da União.

Marôco, J. (2011). Análise estatística com o SPSS statistics. Reportnumber.

Ministério da Educação. (2005). Alunos superdotados terão novas escolas. http://portal.mec.gov.br/ busca-geral/205-noticias/ 1349433645/2529-sp-985633515

Ministério da Educação. (2009). Resolução n. 4. Institui diretrizes operacionais para o atendimento educacional especializado na educação básica, modalidade educação especial. Diário Oficial da União. http://portal.mec. gov.br/dmdocuments/rceb004_09.pdf

Morawska, A., \& Sanders, M. R. (2008). Parenting gifted and talented children: what are the key child behaviour and parenting issues? Australian and New Zealand Journal of Psychiatry, 42(9), 819-827. https://doi.org/ $10.1080 / 00048670802277271$ 
Mudrak, J. (2011). “He was born that way”: Parental constructions of giftedness. High Ability Studies, 22(2), 199-217. https://doi.org/10.1080/13598139.2011.622941

Nakano, T. C., Campos, C. R., \& Santos, M. V. (2016). Escala de avaliação de altas habilidades/superdotação: Versão professor validade de conteúdo. Estudos Interdisciplinares em Psicologia, 7(1), 103-123.

Neihart, M. (2002). Gifted children and depression. In M. Neihart, S. M. Reis, N. M. Robinson, \& S. M. Moon (Eds.), The social and emotional development of gifted children: What do we know? (pp. 930-102). Prufrock.

Neumeister, K. L. S., \& Finch, H. (2006). Perfectionism in high-ability students: Relational precursors and influences on achievement motivation. Gifted Child Quarterly, 50(3), 238-251.https://doi.org/10.1177/001698620605000304

Núcleo de Infância e Família. (2008). Entrevista de dados sociodemográficos da família. Universidade Federal do Rio Grande do Sul.

Ogurlu, Ü., Sevgi-Yalın, H., \& Yavuz-Birben, F. (2016). The relationship between social-emotional learning ability and perceived social support in gifted students. Gifted Education International, 34(1), 76-95. https://doi.org/ $10.1177 / 0261429416657221$

Olszewski-Kubilius, P., Lee, S.-Y., \& Thomson, D. (2014). Family environment and social development in gifted students. Gifted Child Quarterly, 58(3), 199-216. https://doi.org/10.1177/0016986214526430

Ourofino, V. T. A. T., \& Fleith, D. S. (2011). A condição underachievement em superdotação: Definição e características. Psicologia: Teoria e Prática, 13(3), 206-222.

Ourofino, V. T. A. T., \& Guimarães, T. G. (2007). Características intelectuais, emocionais e sociais do aluno com AH/SD. In D. S. Fleith (Ed.), A construção de práticas educacionais para alunos com AH/SD: Orientação a professores (pp. 41-52). MEC; Seesp.

Petersen, J. (2013). Gender differences in identification of gifted youth and in gifted program participation: A meta-analysis. Contemporary Educational Psychology, 38(4), 342-348. https:// doi.org/10.1016/j.cedpsych.2013.07.002

Peterson, J. S. (2015). School counselors and gifted kids: Respecting both cognitive and affective. Journal of Counseling \& Development, 93(2), 153-162. https://doi.org/10.1002/j.1556-6676.2015.00191.x

Pilarinos, V., \& Solomon, C. R. (2017). Parenting styles and adjustment in gifted children. Gifted Child Quarterly, 61(1), 87-98. https://doi.org/10.1177/0016986216675351

Piske, F. H. R. (2013). O desenvolvimento socioemocional de alunos com AH/SD (AH/SD) no contexto escolar: Contribuições a partir de Vygotsky [Dissertação de Mestrado, Universidade Federal do Paraná]. Acervo Digital UFPR. https://acervodigital.ufpr.br/handle/1884/30123?show=full

Piske, F. H. R. (2016). Alunos com AH/SD (AH/SD): Como identificá-los? In H. R. Piske, T. Stoltz, J. M. Machado, \& S. Bahia (Eds.), Altas habilidades/superdotação (AH/SD) e criatividade: Identificação e atendimento (pp. 249-260). Juruá.

Probst, B., \& Piechowski, M. (2012). Overexcitabilities and temperament. In T. L. Cross, \& J. R. Cross (Eds.), Handbook for counselors serving students with gifts and talents (pp. 443-460). Prufrock.

Renati, R., Bonfiglio, N. S., \& Pfeiffer, S. (2017). Challenges raising a gifted child: Stress and resilience factors within the family. Gifted Education International, 33(2), 145-162. https:// doi.org/10.1177/0261429416650948

Richards, J., Encel, J., \& Shute, R. (2003). The emotional and behavioural adjustment of intellectually gifted adolescents: A multi-dimensional, multi-informant approach. High Ability Studies, 14(2), 153-164. http://doi.org/ $10.1080 / 1359813032000163889$

Rocha, M. M. (2012). Evidências de validade do "Inventário de Autoavaliação para Adolescentes" (YSR/2001) para a população brasileira [Tese de doutorado]. Universidade de São Paulo.

Rudasill, K. M., Adelson, J. L., Callahan, C. M., Houlihan, D. V., \& Keizer, B. M. (2013). Gifted students' perceptions of parenting styles: Associations with cognitive ability, sex, race, and age. Gifted Child Quarterly, 57(1), 15-24. https://doi.org/10.1177/0016986212460886

Sapienza, G., Aznar-Farias, M., \& Silvares, E. F. D. M. (2009). Competência social e práticas educativas parentais em adolescentes com alto e baixo rendimento acadêmico. Psicologia:Reflexãoe Crítica, 22(2),208-213.https://doi.org/ $10.1590 /$ S0102-79722009000200006

Semrud-Clikeman, M. (2007). Social competence in children. Springer Science; Business Media. 
Psicologia: Ciência e Profissão 2021 v. 41(n. spe 3), e203883, 1-15.

Shaywitz, S. E., Holahan, J. M., Freudenheim, D. A., Fletcher, J. M., Makuch, R. W., \& Shaywitz, B. A. (2001). Heterogeneity within the gifted: Higher IQ boys exhibit behaviors resembling boys with learning disabilities. Gifted Child Quarterly, 45(1), 16-23. https://doi.org/10.1177/001698620104500103

Silverman, L. K. (2002). Asynchronous development. In M. Neihart, S. M. Reis, N. M. Robinson, \& S. M. Moon (Eds.), The social and emotional development of gifted children: What do we know? (pp. 31-40). Prufrock.

Stoeber, J. (2014). How other-oriented perfectionism differs from self-oriented and socially prescribed perfectionism. Journal of Psychopathology and Behavioral Assessment, 36(2), 329-338. https://doi.org/10.1007/ s10862-013-9397-7

Teixeira, M. A. P., Oliveira, A. M., \&Wottrich, S. H. (2006). Escalas de práticas parentais (EPP): Avaliando dimensões de práticas parentais em relação a adolescentes. Psicologia: Reflexão e Crítica, 19(3), 433-441. http://doi.org/ $10.1590 /$ S0102-79722006000300012

Tentes, V. T. A., \& Fleith, D. S. (2014). Características pessoais, familiares e escolares: Estudo comparativo entre superdotados e superdotados underachievers. Avaliação Psicológica, 13(1), 77-85.

Terrassier, J. C. (2009). Intellectually precocious children. Archives de Pédiatrie, 16(12), 1603-1606. https://doi.org/ 10.1016/j.arcped.2009.07.019

Trentini, C. M., Yates, D. B., \& Heck, V. S. (2014). Escala de Inteligência Wechsler Abreviada (WASI): Manual profissional. Casa do Psicólogo.

Uzunian, L. G., \& Vitalle, M. S. S. (2015). Habilidades sociais: Fator de proteção contra transtornos alimentares em adolescentes. Ciência \& Saúde Coletiva, 20(11), 3495-3508. https://doi.org/10.1590/1413-812320152011.18362014

Valcke, M., Bonte, S., Wever, B., Rots, I. (2010). Internet parenting styles and the impact on internet use of primary school children. Computers \& Education, 55(2), 454-464. https://doi.org/10.1016/j.compedu.2010.02.009

Virgolim, A. M. R. (1997). O indivíduo superdotado: História, concepção e identificação. Psicologia: Teoria e Pesquisa, 13(1), 173-183.

Yazdani, S., \& Daryei, G. (2016). Parenting styles and psychosocial adjustment of gifted and normal adolescents. Pacific Science Review B: Humanities and Social Sciences, 2, 100-105. https://doi.org/10.1016/j.psrb.2016.09.019

\section{Erica Isabel Dellatorre Andrade}

Psicóloga clínica e mestre em psicologia pela Universidade do Vale do Rio dos Sinos (Unisinos), São Leopoldo RS. Brasil.

E-mail: ericadellandrade@gmail.com

(D) https://orcid.org/0000-0002-2737-1157

\section{Jade Wagner Bernardes}

Psicóloga clínica e mestre em Psicologia pela Universidade do Rio Grande do Sul (UFRGS), Porto Alegre - RS. Brasil.

E-mail: jaadebernardes@hotmail.com

(iD) https://orcid.org/0000-0002-1461-5220

\section{Carolina Macedo de Saraiva Lisboa}

Docente no Programa de Pós-Graduação em Psicologia da Pontifícia Universidade Católica do Rio Grande do Sul (PUC-RS), Porto Alegre - RS. Brasil.

E-mail: carolina.lisboa@pucrs.br

(D) https://orcid.org/0000-0002-2199-9824

\section{Angela Helena Marin}

Docente no Programa de Pós-Graduação em Psicologia da Universidade Federal do Rio Grande do Sul, Porto Alegre RS. Brasil.

E-mail: marin.angelah@gmail.com

(D) https://orcid.org/0000-0002-8056-8661 
Artigo derivado da dissertação de mestrado de Erica Isabel Dellatorre Andrade, realizada sob a orientação de Angela Helena Marin e co-orientação de Carolina Macedo de Saraiva Lisboa, apresentada no Programa de Pós-Graduação em Psicologia da Universidade do Vale do Rio dos Sinos.

Endereço para envio de correspondência:

Avenida Diário de Notícias, 200, sala 512, Cristal. CEP: 90810-080. Porto Alegre - RS. Brasil.

Recebido 15/06/2018

Aceito 29/03/2019

Received 06/15/2018

Approved 03/29/2019

Recibido 15/06/2018

Aceptado 29/03/2019

Como citar: Andrade, E. I. D., Bernardes, J. W., Lisboa, C. M. S., \& Marin, A. H. (2021). Práticas educativas parentais e problemas emocionais/comportamentais em adolescentes com altas habilidades/superdotação intelectivas. Psicologia: Ciência e Profissão, 41 (n. spe 3), 1-15. https://doi.org/10.1590/1982-3703003203883

How to cite: Andrade, E. I. D., Bernardes, J. W., Lisboa, C. M. S., \& Marin, A. H. (2021). Parental educational practices and emotional/behavioral problems in high-ability/gifted adolescents. Psicologia: Ciência e Profissão, 41 (n. spe 3), 1-15. https://doi.org/10.1590/1982-3703003203883

Cómo citar: Andrade, E. I. D., Bernardes, J. W., Lisboa, C. M. S., \& Marin, A. H. (2021). Las prácticas educativas parentales y los problemas emocionales/comportamentales en adolescentes con altas habilidades/superdotados. Psicologia: Ciência e Profissão, 41 (n. spe 3), 1-15. https://doi.org/10.1590/1982-3703003203883 